\title{
Uso de residuos agroindustriales en las propiedades mecánicas del concreto: Una revisión literaria
}

Use of agro-industrial waste in the mechanical properties of concrete: A literary review

Uso de resíduos agroindustriais nas propriedades mecânicas do concreto: uma revisão literária

Gustavo Eduardo Castillo Piscoya

cpiscoyagustavo@crece.uss.edu.pe

https://orcid.org/0000-0002-5529-964X

Universidad Señor de Sipán, Chiclayo-Perú

Jorge Keny Peralta Panta

ppantajorgekeny@crece.uss.edu.pe

https://orcid.org/0000-0002-7525-7543

Universidad Señor de Sipán, Chiclayo-Perú
Julio Cesar Chavarry Koosi

ckoosijuli@crece.uss.edu.pe

https://orcid.org/0000-0002-9867-2002

Universidad Señor de Sipán, Chiclayo-Perú

Sócrates Pedro Muñoz Pérez

msocrates@crece.uss.edu.pe

https://orcid.org/0000-0003-3182-8735

Universidad Señor de Sipán, Chiclayo-Perú

Artículo recibido en mayo 2021, revisado en junio 2021, arbitrado en julio 2021 y publicado en septiembre 2021

\section{RESUMEN}

El uso de los residuos agroindustriales se desarrolla dentro del ámbito de la construcción a nivel técnico, ambiental y económico, enfocándose en mejorar la calidad del concreto. Este documento destaca una revisión sistemática de la literatura sobre la aplicación de los residuos agroindustriales en las propiedades mecánicas del concreto, identificando también los porcentajes óptimos y los efectos que estos proporcionan a la mezcla. Se revisaron 62 artículos indexados y distribuidos en las siguientes bases de datos: 34 en Ebsco, 13 en Scielo, 13 en Scopus, y 2 en Science Direct. Concluyendo que, dentro de estos materiales, la mejor opción son las cenizas de centrales térmicas, debido a que resultan ser las más óptimas para ser adicionadas, pues, sus propiedades mecánicas resultaron ser las adecuadas.

Palabras clave: Propiedades mecánicas; Cenizas volantes; Residuo agroindustrial; Concreto; resistencia a la compresión

\section{ABSTRACT}

The use of agro-industrial wastes is developed within the construction field at technical, environmental and economic levels, focusing on improving the quality of concrete. This paper highlights a systematic review of the literature on the application of agroindustrial wastes in the mechanical properties of concrete, identifying also the optimal percentages and the effects they provide to the mix. Sixty-two indexed articles were reviewed and distributed in the following databases: 34 in Ebsco, 13 in Scielo, 13 in Scopus, and 2 in Science Direct. It was concluded that, among these materials, the best option is thermal power plant ashes, since they are the most optimal to be added, as their mechanical properties were found to be adequate.

Key words: Mechanical properties; Fly ash; Agroindustrial waste; Concrete; compressive strength

\section{RESUMO}

O uso de resíduos agro-industriais está se desenvolvendo dentro do campo da construção a nível técnico, ambiental e econômico, com foco na melhoria da qualidade do concreto. Este artigo destaca uma revisão sistemática da literatura sobre a aplicação de resíduos agroindustriais nas propriedades mecânicas do concreto, identificando também as porcentagens ótimas e os efeitos que elas proporcionam à mistura. Sessenta e dois artigos indexados foram revisados e distribuídos nas seguintes bases de dados: 34 em Ebsco, 13 em Scielo, 13 em Scopus, e 2 em Science Direct. Concluiu-se que, entre esses materiais, a melhor opção são as cinzas de usinas térmicas, pois são as mais ideais para serem adicionadas, uma vez que suas propriedades mecânicas foram consideradas adequadas.

Palavras-chave: Propriedades mecânicas; Cinzas volantes; Resíduos agro-industriais; Concreto; Resistência à compressão 


\section{INTRODUCCIÓN}

El incremento del empleo de materiales y tecnologías constructivas convencionales y no convencionales, además de la exigencia de otorgar el desarrollo sostenible en el rubro de la construcción traerá consigo que proyectistas busquen nuevos productos alternativos e innovadores, con características factibles y aceptables para la utilización en la construcción (Krishna, Sandeep, y Mini, 2016). Incluso, a través de diversas investigaciones en todo el mundo se puede corroborar que hoy en día se está requiriendo la utilización de materiales que puedan reemplazar al cemento, tales como: Cenizas volantes, agrícolas escoria y humos de sílice que pueden sustituir adecuadamente al cemento Portland Ordinario (OPC) debido a que tienen propiedades puzolánicas semejantes al material portland, pero con la ventaja que estos productos agroindustriales reducen la contaminación al medio ambiente (Msinjili, Schmidt, Rogge, y Kühne, 2019).

Se puede afirmar que los mayores retos de los cuales confronta la humanidad en la industria de la construcción son: el desarrollo sustentable, la variación del clima, la extracción y el consumo constante de los recursos no renovables (Praveenkumar, Vijayalakshmi, y Meddah, 2019); y basándonos en ello, diversos países se han buscado crear nuevos materiales cementantes que sean innovadores y eficaces, a través del empleo de materias primas alternativas como son los residuos agroindustriales (Katare y Madurwar, 2019).
Hoy en día el concreto es considerado uno de los componentes más empleados en el rubro de la construcción a nivel mundial, puesto que ofrece gran durabilidad, resistencia y versatilidad (Nagajyothi, et al., 2019), sin embargo, debido a que se están presentando nuevas opciones en el rubro ya mencionado, es que están empezando a existir muchos cuestionamientos que ocasiona el uso constante de los materiales que componen al hormigón, específicamente el cemento, dado que, produce problemas medioambientes y de sostenibilidad (Izquierdo, Izquierdo, y Ramalho, 2018).

Ahora, en base a los cuestionamientos que existen debido a las emisiones de $\mathrm{CO} 2$, es que se ha empezado a evaluar la sustitución del cemento portland por los residuos agroindustriales, ya que el cemento es el causante principal que daña al medio ambiente y a la sociedad (Mohd Arif, et al., 2021).

En países como la India, se desperdician cada año aproximadamente 1.60 millones de cascara de huevos, y esto trae consigo que el mal uso de estos residuos agroindustriales genere expulsiones de gas de efecto invernadero que dañan notoriamente al medio ambiente (Sathvik, et al., 2019). Otro de los países en los cuales se está evidenciando este tipo de acontecimientos es Malasia, pues, en el 2017 se ha desechado 85000 toneladas de cascaras de huevo (Tiong, et al., 2020). De igual modo, en Pakistán además de enfrentar los mismos daños ambientales, también se 
ha suscitado problemas energéticos y costos elevados debido al constante uso del cemento, en vez de dar un buen uso a los residuos agroindustriales (Bheel, et al., 2020).

Es imprescindible mencionar en base a los artículos mencionados que desde la antigüedad hasta hoy en día los materiales de construcción han ido pasando diversos procesos, donde se ha demostrado que están surgiendo nuevos materiales innovadores que resultan de los residuos agrícolas, y, por tanto, si a este tipo de desechos se les da un buen uso, surgiría nueva materia prima que al pasar por un cierto proceso surgirían productos capaces de reemplazar o sustituir al cemento.

No obstante, a nivel mundial se están elaborando notables investigaciones con la finalidad de evaluar, identificar y emplear nuevas fuentes alternativas como SCM (Shakouri, et al., 2020). Como, por ejemplo, algunas investigaciones consideran que la reutilización de los residuos agroindustriales para ser incorporaciones en materiales a base de hormigón se está convirtiendo en los últimos años en un tema muy masivo (Tan, Doh, y Chin, 2018). Del mismo modo, han ido tomando mayor relevancia e impacto investigaciones que tratan sobre los efectos que ocasionan este tipo de desechos en materiales como el hormigón, donde se afirma que estos residuos son empleados como sustitución o adición parcial del cemento, para que así, se pueda determinar un porcentaje eficaz (Venkata, Dayakar, y Mohan, 2019).

Cabe precisar, que otros residuos agroindustriales que surgen a partir de la cáscara de arroz, el bagazo de caña de azúcar, también han sido utilizados para sustituir un cierto porcentaje del cemento, que es parte esencial para las mezclas de concreto (Adinna, Nwaiwu, y O Igwagu, 2019). Asimismo, tenemos la mazorca de maíz, sin embargo, son materiales más nobles a comparación de las cenizas volantes (Shakouri, et al., 2020).

Finalmente, en la actualidad se está evaluando la disminución en porcentajes de algunos materiales de construcción, y a su vez se sustituyan por residuos agroindustriales; en razón de, que se ha corroborado como el empleo de dicho producto agroindustrial beneficia en las propiedades mecánicas de los distintos materiales del CM (Berenguer, et al., 2021).

En este sentido, se puede decir que estos materiales agroindustriales están surgiendo como nuevas alternativas capaces de sustituir o reemplazar a los materiales de construcción convencionales, que además de brindar las características deseadas al material que lo conforman, también disminuyen la contaminación y finalmente brindan el desarrollo sustentable en la rama de la ingeniería.

\section{Sobre residuos agroindustriales}

Aunque hay variedad de información acerca de residuos agroindustriales, en todas ellas existe una definición en común: los residuos agrícolas conllevan la disminución de contaminación al medio ambiente y a su vez, el resultado de estas en materiales de construcción. A continuación, se presentan algunas definiciones de residuos agroindustriales, aclarando que se hablaran 
de varios tipos de residuos agrícolas desde la construcción, como un campo de conocimiento o de aplicación. En este sentido, se realizó una revisión general de las teorías de residuos agroindustriales.

Para Šadzevičius, Gurskis, y Ramukevičius (2015)indicanque:"Construction has notalways been based on caring for the environment by using some type of material". En base a esta definición, se puede mencionar que en pleno S.XXI el mercado de la construcción (CM) está pasando por un proceso sustentable, el cual no siempre ha generado un aspecto favorable para el medio ambiente, pues, se ha estado centrando en buscar materiales innovadores, eficaces, sin tomar en cuenta si son respetuoso con el medio ambiente. Por su parte Alengaram, et al., (2016), presentan que a nivel mundial el aumento de la demanda en el CM ha conllevado el excesivo uso del hormigón; y a su vez el empleo constante de este tipo de materiales convencionales que daña considerablemente al ambiente y a la sociedad, puesto que los materiales que lo componen no serían considerados materiales respetuosos con el medio ambiente. Todo esto, está originando que se busquen nuevas alternativas eficaces, resistentes y favorables para que sean empleados en los materiales del CM, como la fabricación del concreto. Luego se ha presenciado que con el surgimiento de estos nuevos productos innovadores están acelerando en el CM su desarrollo. $Y$ esto también se relaciona con: los productos agrícolas, residuos agroindustriales y la capacidad para formar productos novedosos. No obstante, con la velocidad que se podrían formar estos productos está llevando a seguir realizando diversos estudios, dejando en evidencia lo necesario que sería innovar con los residuos agroindustriales.

Jannat, et al., (2020) vincula el proceso de producción de materiales del CM con la gran demanda de energía que esto conlleva, señalando que: Una definición general respecto al proceso de fabricación de materiales de construcción es genera un consumo de energía excesivo que perjudica al medio ambiente. Asimismo, este autor indica que "el empleo de materiales de construcción en base a residuos agrícolas beneficia notoriamente al CM y al medio ambiente, y por ende no necesitamos hacer uso excesivo de energía, además que hacer uso de este tipo de residuos ayudaría a crear nuevos productos innovadores". Igualmente, se hace énfasis en la importancia del uso de residuos agroindustriales para elaborar materiales de construcción que tengan buenas "physicalmechanical characteristics" (Townsend, et al., 2020).

Adjei y Elkatatny (2020) explica que "la principal fuente de expulsión de $\mathrm{CO} 2$ es por la producción del cemento, siendo este el producto principal para la elaboración del concreto". Por el contrario, en cuanto a desarrollo sostenible Charitha, et al., (2021) sostiene que: "los productos alternativos en base a residuos agroindustriales serían los más adecuados para el sector construcción que pueden ser empleados en la elaboración del hormigón". En este sentido, se pone de evidencia que los residuos agroindustriales promueven el desarrollo productos innovadores y sostenibles. 
La utilización de los residuos agroindustriales para la fabricación de materiales de construcción tales como concreto, son un beneficio para el CM, puesto que beneficia en el desarrollo sostenible para el medio ambiente, dando una solución a la constante contaminación por los desechos sólidos (Katare y Madurwar, 2021). Asimismo Clark, et al., (2017), menciona que se ha comprobado gracias a diversas investigaciones que la utilización de residuos agroindustriales en la elaboración de los materiales de construcción está resultando ser un éxito, puesto que sus diferentes características están siendo las esperadas.

Ahora, para Athira, et al., (2021) define la aplicación de residuos agrícolas en materiales de construcción como elementos sostenibles que ayudarían a disminuir el uso de recursos naturales y energía y, a su vez, permitiría que se haga un buen uso, puesto que este tipo de residuos sólidos generan preocupación en diversos países de desarrollo. Además, (Mishurov, et al., 2021) menciona que el uso de residuos provenientes de la agricultura son lo suficientemente capaces para ser empleados en la elaboración de materiales constructivos como el concreto, demostrando así que se pueden generar productos modernos a partir de los residuos agroindustriales.

De igual modo, Memon, et al., (2021) hace referencia que el uso de SCM a base de desechos agroindustriales se sigue incrementando conforme pasan los años, evidenciando una vez más que estos productos nuevos e innovadores tienen buen desempeño al ser incorporados en materiales tales como el concreto, y pueden ayudar a disminuir el uso del cemento, que con su uso constante genera fuertes emisiones de $\mathrm{CO} 2$, afectando así al medio ambiente.

En base a los artículos mencionado se puede decir que el uso adecuado de los residuos agroindustriales beneficiaria al CM, puesto que son residuos sólidos que disminuyen el consumo de energía, la contaminación ambiental y permitiría la reducción de costos.

\section{Alcance delusoderesiduosagroindustriales en el concreto}

Se ha demostrado que materiales como el hormigón están siendo los más empleados, en el sector de infraestructura y construcción, debido a que son capaces de mejorar la propiedades tanto físicas - mecánicas del material (Scope, Vogel, y Guenther, 2021). No obstante, a pesar de la popularidad que hoy en día tiene por las buenas y eficaces características también existe un alto indicador negativo respecto a la producción de estos materiales, tales son: Los morteros $u$ hormigones, debido a que, para su elaboración es necesario el cemento; y ello conlleva el uso constante de este recurso natural que demanda mucha energía, sin dejar de lado las expulsiones en grandes cantidades de $\mathrm{CO} 2$ (Herrmann, Koenig, y Dehn, 2018).

En países de Sudamérica, desde el 2018 han considerado que se realice el aprovechamiento de los desechos que permitan incentivar al reciclaje. Sin embargo, no se ha logrado cumplir con las expectativas, pese a que se ha comprobado en distintas investigaciones que cuando estos residuos 
agroindustriales son bien aprovechados se pueden obtener subproductos con características y propiedades eficaces que pueden ser utilizados en el CM (Peñaranda, Montenegro y Giraldo, 2017).

Las mejoras constantes en los materiales que componen al hormigón han permitido que en la actualidad se empiece hacer uso de residuos agroindustriales con la finalidad de disminuir la contaminación al medio ambiente, pero a su vez, brindar a este material buenas características física-mecánicas.

El uso de los residuos agroindustriales, a partir de las cenizas de caña de azúcar han resultado una posible alternativa sostenible para ser aplicado en la elaboración del concreto, puesto que, algunas investigaciones evidenciaron que las propiedades mecánicas del material ya mencionado han sido los deseados Minnu y Bahurudeen, (2020). Incluso, emplear este tipo de residuos que se generan a partir de productos agrícolas, es beneficioso, debido a que son materiales con alta disponibilidad y no requiere de mayores costos para que puedan ser adquiridos y utilizados en la elaboración de diversos materiales del CM (Athira, Bahurudeen, y Vishnu, 2021).

Badiale Furlong, et al., (2020) el área que abarca la construcción civil permite aprovechar adecuadamente los recursos agroindustriales a través del uso de las siguientes cenizas: de cáscara de arroz, del bagazo de caña de azúcar, de cáscara de huevo y de mazorca de maíz en la mezcla del concreto, que conllevan a la reducción de materiales que de alguna manera u otra dañan al medio ambiente. Ahora, dichos materiales o residuos agrícolas han demostrado la gran capacidad de reacción al ser sustituidos en materiales como morteros u hormigones.

Como se sabe, el CM ha ocasionado durante muchos años, incluso en la actualidad la extracción de los recursos naturales, y todo esto genera una constante extracción de recursos naturales acompañado de una gran fuente de energía. Por tal, es que, a partir de ese aspecto, surge la necesidad de la fabricar nuevos sub-productos innovadores y eficaces para que así se reduzca los impactos ambientales, pero sin dejar de lado que cumplan con las expectativas al sustituirse en los materiales de construcción (Souza, y otros, 2021).

Hay aspectos importantes que se toman en cuenta para la realización de los productos de hormigón, y uno de ellos es: poseer buenas propiedades tanto físicas y mecánicas que los conviertan en productos capaces de ser empleados en la construcción. Basándose, en lo ya mencionado, algunas investigaciones están hoy en día empleando nuevos productos agroindustriales con el beneficio de cumplir cada una de las expectativas (Jagadesh, Ramachandramurthy, y Murugesan, 2018). Del mismo modo (Rodier, Villar Cociña, Ballesteros, y Holmer Savastano, 2019), considera que la fabricación de productos de constructivos con adiciones de residuos agroindustriales sería beneficioso, debido a que sus costos serían menores.

Además, dentro de los residuos agroindustriales, también tenemos el uso de las cenizas que son generadas a partir de residuos agrícolas, ya que, diversas investigaciones 
donde fueron utilizadas las cenizas de bagazo de caña de azúcar en la mezcla del concreto, obtuvieron como resultado que sus propiedades mecánicas fueron las más adecuadas (Zareei, Ameri, y Bahrami, 2018). Por tal, basándose en el uso de los residuos agroindustriales, se está considerando conveniente fabricarse estos residuos a partir de productos agrícolas, debido a que mejora notoriamente el desempeño mecánico (Hernández, et al., 2020). Por otro, se debe destacar que las cenizas volantes empleado en la elaboración de alta resistencia se está convirtiendo en un tema muy investigado; ya que, ha demostrado un alto porcentaje de su resistencia a la compresión durante los últimos días de curado, con adiciones de un 40\% (Aggarwal, Gupta, y Sachdeva, 2015).

En efecto, el presente artículo busca analizar y dar el conocer el beneficio que trae consigo el uso de los residuos agroindustriales para las propiedades mecánicas del hormigón, siendo éste un material innovador, sostenible, y con alta disponibilidad.

\section{MÉTODO}

Debido a la variedad de residuos agroindustriales que en la actualidad se han usado como parte de la solución del problema planteado, la presente revisión sistemática solo tratará de los siguientes residuos agroindustriales: 1) la ceniza de cáscara de arroz, 2) la ceniza de cáscara de huevo, 3) ceniza de bagazo de caña de azúcar, 4) cenizas de centrales térmicas, 5) cenizas volantes. Teniendo como punto principal los valores de resistencia a la compresión del concreto.

La revisión se realizó con 70 artículos indexados distribuidos de la siguiente manera: 8 artículo en el año 2015, 4 en el año 2016, 3 en el año 2017, 9 en el año 2018, 15 en el año 2019, 18 en el año 2020 y 13 artículos en el año 2021. Para la búsqueda de los artículos se usaron las siguientes palabras clave: Fly ash, agro-industrial waste, mechanical properties, concrete, use of agro-industrial waste in construction, use of residues from egg hull ash and corn masorca in construction, what is concrete as a construction material, comparative tables of the mechanical properties of concrete, what benefit does the use of agroindustrial waste in construction generate?

A continuación, para un mejor detalle: 
Tabla 1. Criterios de búsqueda según base de datos, palabras clave y año de publicación.

\begin{tabular}{|c|c|c|c|c|c|c|}
\hline \multirow{2}{*}{$\begin{array}{c}\text { Base de } \\
\text { datos }\end{array}$} & \multirow[b]{2}{*}{ Palabras clave } & \multirow{2}{*}{$\begin{array}{l}\text { Sin filtro } \\
\text { Resultado }\end{array}$} & \multicolumn{2}{|c|}{ Aplicación de filtros } & \multicolumn{2}{|c|}{ Resultados } \\
\hline & & & Año & Otros & $\begin{array}{l}\text { Documentos } \\
\text { encontrados }\end{array}$ & $\begin{array}{l}\text { Documentos } \\
\text { seleccionados }\end{array}$ \\
\hline \multirow[t]{4}{*}{ EBSCO } & $\begin{array}{c}\text { Use of } \\
\text { agro-industrial } \\
\text { waste in } \\
\text { construction }\end{array}$ & 36189 & $\begin{array}{l}2015- \\
2021\end{array}$ & $\begin{array}{c}\text { Materia: Término del } \\
\text { tesauro } \\
\text {-Agricultural ecology } \\
\text {-Agricultural } \\
\text { industries } \\
\text {-Agriculture } \\
\text {-Industrial wastes } \\
\text {-Sustainable } \\
\text { agriculture }\end{array}$ & 2030 & 24 \\
\hline & $\begin{array}{c}\text { What is concrete } \\
\text { as a construction } \\
\text { material }\end{array}$ & 2 & --- & --- & 2 & 1 \\
\hline & $\begin{array}{l}\text { Comparative } \\
\text { tables of the } \\
\text { mechanical } \\
\text { properties of } \\
\text { concrete }\end{array}$ & 33486 & $\begin{array}{l}2015- \\
2021\end{array}$ & $\begin{array}{c}\text { Materia: Término del } \\
\text { tesauro } \\
\text {-comparative studies } \\
\text {-concrete } \\
\text {-construction } \\
\text { materials } \\
\text {-mechanical behavior } \\
\text { of materials } \\
\text {-compressive strength } \\
\text {-composite materials } \\
\text {-cement }\end{array}$ & 1937 & 18 \\
\hline & $\begin{array}{l}\text { Comparative } \\
\text { tables of the } \\
\text { mechanical } \\
\text { properties of } \\
\text { concrete }\end{array}$ & 33486 & $\begin{array}{l}2015- \\
2021\end{array}$ & $\begin{array}{c}\text { Materia: Término del } \\
\text { tesauro } \\
\text {-comparative studies } \\
\text {-concrete } \\
\text {-construction } \\
\text { materials } \\
\text {-mechanical behavior } \\
\text { of materials } \\
\text {-compressive strength } \\
\text {-composite materials } \\
\text {-cement }\end{array}$ & 1937 & 18 \\
\hline \multirow[t]{2}{*}{ SCOPUS } & $\begin{array}{l}\text { Use of agro- } \\
\text { industrial waste in } \\
\text { construction }\end{array}$ & 66 & $\begin{array}{l}2015- \\
2021\end{array}$ & $\begin{array}{c}\text { Subject area: } \\
\text {-Agricultural and } \\
\text { Biological Sciences } \\
\text {-Energy } \\
\text {-Engineering } \\
\text {-Environmental } \\
\text { Science } \\
\text {-Materials Science }\end{array}$ & 43 & 7 \\
\hline & $\begin{array}{c}\text { What is concrete } \\
\text { as a construction } \\
\text { material }\end{array}$ & 409 & $\begin{array}{l}2015- \\
2021\end{array}$ & $\begin{array}{l}\text { Subject area: } \\
\text {-Engineering } \\
\text {-Material Science } \\
\text {-Environmental } \\
\text { Science } \\
\text { Energy }\end{array}$ & 137 & 12 \\
\hline
\end{tabular}




\begin{tabular}{|c|c|c|c|c|c|c|}
\hline \multirow{2}{*}{$\begin{array}{l}\text { Base de } \\
\text { datos }\end{array}$} & \multirow[b]{2}{*}{ Palabras clave } & \multirow{2}{*}{$\begin{array}{l}\text { Sin filtro } \\
\text { Resultado }\end{array}$} & \multicolumn{2}{|c|}{ Aplicación de filtros } & \multicolumn{2}{|c|}{ Resultados } \\
\hline & & & Año & Otros & $\begin{array}{l}\text { Documentos } \\
\text { encontrados }\end{array}$ & $\begin{array}{l}\text { Documentos } \\
\text { seleccionados }\end{array}$ \\
\hline SCIELO & $\begin{array}{l}\text { Cement, } \\
\text { fly-ash, } \\
\text { concrete, } \\
\text { design. }\end{array}$ & 2 & --- & --- & 2 & 1 \\
\hline \multirow[t]{4}{*}{$\begin{array}{l}\text { SCIENCE } \\
\text { DIRECT }\end{array}$} & $\begin{array}{l}\text { Rice husk ash, } \\
\text { amorphous } \\
\text { material, cement } \\
\text { manufacture, } \\
\text { compressive } \\
\text { strength, } \\
\text { Pozzolan }\end{array}$ & 378 & $\begin{array}{l}2015- \\
2021\end{array}$ & $\begin{array}{c}\text { Subject areas: } \\
\text {-Engineering } \\
\text {-Materials Science } \\
\text {-Environmental } \\
\text { Science } \\
\text {-Agricultural and } \\
\text { Biological Sciences }\end{array}$ & 243 & 1 \\
\hline & $\begin{array}{l}\text { Supplementary } \\
\text { cementitious } \\
\text { materials } \\
\text { Corn cob ash } \\
\text { Cement hydration } \\
\text { Concrete } \\
\text { Durability }\end{array}$ & 61 & $\begin{array}{l}2015- \\
2021\end{array}$ & $\begin{array}{c}\text { Subject areas: } \\
\text {-Engineering } \\
\text {-Materials Science } \\
\text {-Environmental } \\
\text { Science }\end{array}$ & 50 & 1 \\
\hline & $\begin{array}{c}\text { Concrete, rice husk } \\
\text { ashes }\end{array}$ & 1042 & $\begin{array}{l}2015- \\
2021\end{array}$ & $\begin{array}{l}\text { Subject areas: } \\
\text {-Engineering } \\
\text {-Materials Science }\end{array}$ & 566 & 4 \\
\hline & $\begin{array}{l}\text { Cane bagasse ash } \\
\text { in concrete }\end{array}$ & 144 & $\begin{array}{l}2015- \\
2021\end{array}$ & $\begin{array}{c}\text { Subject areas: } \\
\text {-Engineering } \\
\text {-Materials Science }\end{array}$ & 99 & 1 \\
\hline
\end{tabular}

Tabla 2. Artículos por año de publicación.

\begin{tabular}{|c|c|c|c|c|c|c|c|c|}
\hline \multirow{2}{*}{$\begin{array}{l}\text { BASE DE } \\
\text { DATOS }\end{array}$} & \multicolumn{7}{|c|}{ AÑO DE PUBLICACIÓN } & \multirow{2}{*}{ TOTAL } \\
\hline & 2015 & 2016 & 2017 & 2018 & 2019 & 2020 & 2021 & \\
\hline EBSCO & 8 & 3 & 3 & 7 & 11 & 13 & 6 & 51 \\
\hline SCOPUS & & 1 & & 2 & 4 & 2 & 4 & 13 \\
\hline SCIENCE DIRECT & & & & & & 3 & 2 & 5 \\
\hline SCIELO & & & & & & & 1 & 1 \\
\hline
\end{tabular}


Tabla 3. Uso de los diferentes residuos agroindustriales para la elaboración de materiales a base de concreto en diferentes países.

\section{RESIDUO AGROINDUSTRIAL}

Ceniza de cáscara de arroz

Ceniza de escoria de alto horno

Cenizas volantes

Ceniza de hoja de maíz y bagazo de caña

PAISES DONDE LO EMPLEARON

España, Perú, Argentina, Habana Taiwan, Egipto, Arabia Saudita, Pakistan

Colombia, España, Argentina.

España.

Bucaramanga, Colombia, Brazil.

Venezuela, Pakistan, India, España

Panamá, Kenya, Malasia.

\section{REFERENCIAS}

(Cabrera, Sánchez Rodríguez, y Aballí Altamirano, 2015), (Celik, Meral, \& Monteiro, 2015), (Mattey, Robayo, Díaz, y Delvasto, 2015), (Fang-Chen, Ming-Gin, \& Shang-Lien, 2020), (A. Serag, Sahar A., Bassam A., y Taher A., 2021), (Bassam A., Rayed, Hisham, y Abdulaziz, 2020). (Tariq, Abdullah, Daddan Khan, Abdul Salam, y Zaheer, 2021)

(Montiel Bohórquez y Pérez, 2019), (Vargas y Pérez, 2018), (Huaquisto y Belizario, 2018)

(Fuentes, Fragozo, y Vizcaino, 2015)

(Gonzáles, Montaño, y Castro, 2015), (Prakash, Thenmozhi, y Raman, 2020), (Fernández y Palomo, 2015), (D., D. Marcon, C., A., y G. A., 2020)

(Águila y Sosa, 2008), (Da Silva, Basto, Araujo, Miranda, y Neto, 2019), (Farnaz, Arjumend, y Mehmood, 2020), (Rajasekar, Arunachalam, Kottaisamy, y Saraswathy , 2018), (Torres de Sande, y otros, 2021)

(Aizpurúa, Moreno, y Caballero, 2018), (Oliko, Kabubo, \& Mwero, 2020), (Ashraf y SI , 2020) 


\section{DESARROLLO Y DISCUSIÓN}

Tabla 4. Resistencia a compresión del concreto con los diferentes residuos agroindustriales..

\begin{tabular}{|c|c|c|c|c|c|c|}
\hline $\begin{array}{l}\text { RESIDUO } \\
\text { AGRO- } \\
\text { INDUSTRIAL }\end{array}$ & $\begin{array}{c}\text { SUSTITUCIÓN } \\
(\%)\end{array}$ & $\begin{array}{l}\text { DÍAS DE } \\
\text { CURADO }\end{array}$ & $\begin{array}{l}\text { Cont. de } \\
\text { cemento } \\
\left(\mathrm{kg} / \mathrm{m}^{3}\right)\end{array}$ & $a / c$ & $\begin{array}{c}F^{\prime} C \\
\left(\mathrm{~kg} / \mathrm{cm}^{2}\right)\end{array}$ & REFERENCIA \\
\hline \multirow{29}{*}{$\begin{array}{l}\text { Cenizas de } \\
\text { cáscara de } \\
\text { huevo }\end{array}$} & \multirow[t]{2}{*}{ MP } & 7 & \multirow[t]{2}{*}{720} & \multirow[t]{8}{*}{0.32} & 470 & \multirow{8}{*}{$\begin{array}{c}\text { (Mohd Arif, } \\
\text { y otros, 2021) }\end{array}$} \\
\hline & & 28 & & & 671 & \\
\hline & \multirow[t]{2}{*}{5} & 7 & \multirow[t]{2}{*}{684} & & 477 & \\
\hline & & 28 & & & 681 & \\
\hline & \multirow[t]{2}{*}{10} & 7 & \multirow[t]{2}{*}{648} & & 479 & \\
\hline & & 28 & & & 684 & \\
\hline & \multirow[t]{2}{*}{15} & 7 & \multirow[t]{2}{*}{612} & & 437 & \\
\hline & & 28 & & & 625 & \\
\hline & \multirow[t]{3}{*}{ MP } & 7 & \multirow[t]{3}{*}{400} & \multirow[t]{15}{*}{0.45} & 220 & \multirow{15}{*}{$\begin{array}{c}\text { (Tan, Doh, } \\
\text { y Chin, 2018) }\end{array}$} \\
\hline & & 14 & & & 260 & \\
\hline & & 28 & & & 310 & \\
\hline & \multirow[t]{3}{*}{5} & 7 & \multirow[t]{3}{*}{380} & & 330 & \\
\hline & & 14 & & & 370 & \\
\hline & & 28 & & & 420 & \\
\hline & \multirow[t]{3}{*}{10} & 7 & \multirow[t]{3}{*}{360} & & 400 & \\
\hline & & 14 & & & 420 & \\
\hline & & 28 & & & 460 & \\
\hline & \multirow[t]{3}{*}{15} & 7 & \multirow[t]{3}{*}{340} & & 410 & \\
\hline & & 14 & & & 450 & \\
\hline & & 28 & & & 490 & \\
\hline & \multirow[t]{3}{*}{20} & 7 & \multirow[t]{3}{*}{320} & & 350 & \\
\hline & & 14 & & & 360 & \\
\hline & & 28 & & & 370 & \\
\hline & \multirow[t]{3}{*}{ MP } & 7 & \multirow[t]{6}{*}{ NR } & \multirow[t]{6}{*}{$N R$} & 109.2 & \multirow{6}{*}{$\begin{array}{l}\text { (Aizpurúa, Moreno, } \\
\text { y Caballero, 2018) }\end{array}$} \\
\hline & & 14 & & & 141.2 & \\
\hline & & 28 & & & 164.6 & \\
\hline & \multirow[t]{3}{*}{1.5} & 7 & & & 106.1 & \\
\hline & & 14 & & & 142.5 & \\
\hline & & 28 & & & 172.5 & \\
\hline
\end{tabular}




\begin{tabular}{|c|c|c|c|c|c|c|}
\hline $\begin{array}{c}\text { RESIDUO } \\
\text { AGRO- } \\
\text { INDUSTRIAL } \\
\end{array}$ & $\begin{array}{c}\text { SUSTITUCIÓN } \\
(\%)\end{array}$ & $\begin{array}{l}\text { DÍAS DE } \\
\text { CURADO }\end{array}$ & $\begin{array}{c}\text { Cont. de } \\
\text { cemento } \\
\left(\mathrm{kg} / \mathrm{m}^{3}\right)\end{array}$ & $a / c$ & $\begin{array}{c}F^{\prime} \mathrm{C} \\
\left(\mathrm{kg} / \mathrm{cm}^{2}\right)\end{array}$ & REFERENCIA \\
\hline \multirow{20}{*}{$\begin{array}{c}\text { Cenizas de } \\
\text { cáscara de arroz }\end{array}$} & \multirow[t]{3}{*}{ MP } & 7 & NR & $N R$ & 268 & \multirow{12}{*}{$\begin{array}{c}\text { (Camargo y Higuera, } \\
\text { 2017) }\end{array}$} \\
\hline & & 14 & & & 328.4 & \\
\hline & & 28 & & & 346.4 & \\
\hline & \multirow[t]{3}{*}{5} & 7 & & & 302.8 & \\
\hline & & 14 & & & 314.9 & \\
\hline & & 28 & & & 351.7 & \\
\hline & \multirow[t]{3}{*}{15} & 7 & & & 150.3 & \\
\hline & & 14 & & & 183.2 & \\
\hline & & 28 & & & 188.6 & \\
\hline & \multirow[t]{3}{*}{30} & 7 & & & 62.5 & \\
\hline & & 14 & & & 108.6 & \\
\hline & & 28 & & & 106.4 & \\
\hline & \multirow[t]{4}{*}{ MP } & 7 & 400 & 0.45 & 320 & \multirow{8}{*}{$\begin{array}{l}\text { (Robayo, Mattey, } \\
\text { y Delvasto, 2015) }\end{array}$} \\
\hline & & 14 & & & 360 & \\
\hline & & 28 & & & 405 & \\
\hline & & 60 & & & 445 & \\
\hline & \multirow[t]{4}{*}{20} & 7 & \multirow[t]{4}{*}{320} & & 230 & \\
\hline & & 14 & & & 270 & \\
\hline & & 28 & & & 350 & \\
\hline & & 60 & & & 42.5 & \\
\hline \multirow{10}{*}{$\begin{array}{l}\text { Ceniza de } \\
\text { bagazo de caña } \\
\text { de azúcar }\end{array}$} & \multirow[t]{2}{*}{ MP } & 7 & \multirow[t]{6}{*}{ NR } & \multirow[t]{6}{*}{$N R$} & 144.25 & \multirow{6}{*}{$\begin{array}{c}\text { (Ruiz, Peñaranda, } \\
\text { Fuentes, y Semprun, } \\
\text { 2020) }\end{array}$} \\
\hline & & 28 & & & 212.75 & \\
\hline & 20 & 7 & & & 140 & \\
\hline & & 28 & & & 162.75 & \\
\hline & \multirow[t]{2}{*}{40} & 7 & & & 177.75 & \\
\hline & & 28 & & & 162.5 & \\
\hline & MP & NR & 10.26 & $N R$ & 353.55 & \\
\hline & 5 & NR & & & 305.9 & \\
\hline & 10 & NR & & & 268.09 & \\
\hline & 20 & NR & & & 179.37 & \\
\hline
\end{tabular}




\begin{tabular}{|c|c|c|c|c|c|c|}
\hline $\begin{array}{l}\text { RESIDUO } \\
\text { AGRO- } \\
\text { INDUSTRIAL }\end{array}$ & $\begin{array}{l}\text { SUSTITUCIÓN } \\
\text { (\%) }\end{array}$ & $\begin{array}{l}\text { DÍAS DE } \\
\text { CURADO }\end{array}$ & $\begin{array}{l}\text { Cont. de } \\
\text { cemento } \\
\left(\mathrm{kg} / \mathrm{m}^{3}\right)\end{array}$ & $a / c$ & $\begin{array}{c}F^{\prime} \mathrm{C} \\
\left(\mathrm{kg} / \mathrm{cm}^{2}\right)\end{array}$ & REFERENCIA \\
\hline \multirow{20}{*}{$\begin{array}{l}\text { Cenizas de } \\
\text { centrales } \\
\text { térmicas }\end{array}$} & \multirow[t]{3}{*}{ MP } & 7 & \multirow[t]{3}{*}{42.5} & \multirow[t]{15}{*}{0.56} & 146 & \multirow{15}{*}{$\begin{array}{c}\text { (Huaquisto y Belizario, } \\
\text { 2018) }\end{array}$} \\
\hline & & 14 & & & 178 & \\
\hline & & 28 & & & 218 & \\
\hline & \multirow[t]{3}{*}{2.5} & 7 & \multirow[t]{3}{*}{41.44} & & 147 & \\
\hline & & 14 & & & 180 & \\
\hline & & 28 & & & 223 & \\
\hline & \multirow[t]{3}{*}{5} & 7 & \multirow[t]{3}{*}{40.38} & & 150 & \\
\hline & & 14 & & & 185 & \\
\hline & & 28 & & & 231 & \\
\hline & \multirow[t]{3}{*}{10} & 7 & \multirow[t]{3}{*}{38.25} & & 139 & \\
\hline & & 14 & & & 170 & \\
\hline & & 28 & & & 200 & \\
\hline & \multirow[t]{3}{*}{15} & 7 & \multirow[t]{3}{*}{36.13} & & 125 & \\
\hline & & 14 & & & 159 & \\
\hline & & 28 & & & 192 & \\
\hline & MP & NR & 50 & \multirow[t]{5}{*}{ NR } & 164 & \multirow{5}{*}{$\begin{array}{c}\text { (Ballesteros, Serrano, } \\
\text { y Pérez, 2019) }\end{array}$} \\
\hline & 10 & NR & 45 & & 115 & \\
\hline & 20 & NR & 40 & & 124 & \\
\hline & 30 & NR & 35 & & 103 & \\
\hline & 40 & NR & 30 & & 113 & \\
\hline \multirow{18}{*}{$\begin{array}{l}\text { Cenizas } \\
\text { volantes }\end{array}$} & \multirow[t]{2}{*}{ MP } & 28 & \multirow[t]{2}{*}{410} & \multirow[t]{6}{*}{0.5} & 329.4 & \multirow{6}{*}{$\begin{array}{l}\text { (Canul, Moreno, } \\
\text { y Mendoza, 2016) }\end{array}$} \\
\hline & & 91 & & & 360.6 & \\
\hline & \multirow[t]{2}{*}{20} & 28 & \multirow[t]{2}{*}{324.7} & & 300 & \\
\hline & & 91 & & & 328.4 & \\
\hline & \multirow[t]{2}{*}{40} & 28 & \multirow[t]{2}{*}{241} & & 232.9 & \\
\hline & & 91 & & & 272.9 & \\
\hline & \multirow[t]{4}{*}{ MP } & 7 & \multirow[t]{4}{*}{550} & NR & 288 & (Silva y Delvasto, 2015) \\
\hline & & 14 & & & 339 & \\
\hline & & 28 & & & 384 & \\
\hline & & 90 & & & 527 & \\
\hline & 35 & 7 & 357.5 & & 289 & \\
\hline & & 14 & & & 351 & \\
\hline & & 28 & & & 434 & \\
\hline & & 90 & & & 489 & \\
\hline & 50 & 7 & 275 & & 245 & \\
\hline & & 14 & & & 316 & \\
\hline & & 28 & & & 349 & \\
\hline & & 90 & & & 465 & \\
\hline
\end{tabular}




\section{DISCUSIÓN}

\section{La ceniza de cascarilla de arroz}

(Camargo y Higuera, 2017) menciona que, al aumentar el porcentaje de sustitución de CCA en la mezcla de concreto, la resistencia a la compresión tiende a disminuir. De igual manera resultaron las investigaciones de (Robayo, Mattey, y Delvasto, 2015), el cual indica que al sustituir el $20 \%$ también disminuye la resistencia a la compresión, presentando su mayor declive a los 60 días.

\section{La ceniza de cáscara de huevo}

Una sustitución óptima en proporciones de 1.5 h (cenizas de cáscara de huevo) brinda un incremento de resistencia en $4.8 \%$ en comparación a la mezcla sin sustitución de ceniza (SC) a los 28 días (Aizpurúa, Moreno, y Caballero, 2018). Además, en la Tabla 5 , se observan los valores de los ensayos realizados a $\operatorname{los} 7,14,28$ días, donde se verifica que a edades tempranas los residuos agroindustriales adicionados no aportan en la resistencia del concreto. Sin embargo, en los días de curado posteriores ocurre lo contrario, puesto que se ve un incremento en su resistencia a la compresión.

En los resultados de (Tan, Doh, y Chin, 2018) nos indican que el hormigón con cáscara de huevo mejoró en su resistencia a la compresión conforme se aumentaba la sustitución del material agroindustrial. Se puede observar hasta un $58.1 \%$ de incremento en la resistencia a la compresión a los 28 días con una sustitución del 15\% de CCA.
Mohd Arif, et al., (2021) realizaron su investigación sustituyendo ceniza de cáscara de huevo en porcentajes de 5, 10 y $15 \%$, llegando a la conclusión de que el porcentaje óptimo para obtener la más alta resistencia a la compresión es de $10 \%$, tanto en 7 como a los 28 días de curado. Además, presenta una disminución de trabajabilidad a medida que el porcentaje de reemplazo va aumentando, demostrando que el material tiene potencial para ser utilizado en la construcción.

\section{La ceniza de bagazo de caña de azúcar}

Ruiz, et al., (2020) menciona que su resistencia a la compresión inicial (7 días de curado) alcanza su valor máximo con una sustitución del $40 \%$, mientras que, a los 28 días, la resistencia disminuye al incrementar el porcentaje de sustitución.

Elías, Sichez, y Reyna, (2019) evidencian que conforme fue mayor el porcentaje de ceniza de bagazo de caña de azúcar, disminuye la resistencia del concreto, demostrando que dicha ceniza no es un material adecuado para la construcción.

\section{La ceniza de centrales térmicas}

Huaquisto y Belizario (2018) realizaron su investigación con 4 porcentajes de sustitución, los cuales fueron: 2.5, 5, 10 y $15 \%$ con una relación agua/cemento igual 0.56 , dejando demostrado que, a los 7, 14 y 28 días, la resistencia alcanza su valor más alto al sustituirle $5 \%$ de cenizas, sin embargo, mientras se aumenta el porcentaje, la resistencia a la compresión disminuye. De igual manera, (Ballesteros, Serrano, y Pérez, 2019) coinciden 
con los resultados de (Huaquisto y Belizario, 2018), demostrando que, a mayor aumento de porcentaje de sustitución de cenizas de centrales térmicas, la resistencia a la compresión disminuye.

\section{Las cenizas volantes}

Canul, Moreno,y Mendoza, 2016)Considera que la mezcla de concreto con cenizas no permite una mejora en su resistencia a la compresión; pues se observó que con la sustitución de $20 \%$ y $40 \%$ su resistencia disminuyó. En cambio (Silva y Delvasto, 2015), afirma que las cenizas volantes mejoran las propiedades de la mezcla al sustituirse el $35 \%$ del material, obteniendo un aumento de hasta $13 \%$ de la resistencia a la compresión en el último día de curado. Por otra parte, al sustituir el $50 \%$ de la mezcla, su resistencia comienza a disminuir.

\section{CONCLUSIÓN}

Mediante el análisis del estado del arte en la temática se concluye que la incorporación de residuos agroindustriales en el concreto logra:

La sustitución de las cenizas de cáscaras de huevo permite el incremento de la resistencia a la compresión con $1.5 \mathrm{~h} \%$ puesto que es un material con gran porcentaje óxido de calcio, el cual es el responsable de la formación de los compuesto cementantes. No obstante, también se ha comprobado que la ceniza de la cáscara de huevo no sería un material adecuado cuando se emplea en determinados porcentajes, debido a que se experimenta una reducción de trabajabilidad en valores de $30-70 \%$ y su resistencia a la compresión se disminuye en un $13-48 \%$ con respecto al testigo patrón.

Adicionar en un 5\% de CCA favorece la resistencia a la compresión del concreto, puesto que lo convierte en un compuesto viable para la elaboración de materiales de construcción.

Se destaca el efecto positivo de la utilización de los residuos agroindustriales, puesto que su aplicación conllevaría a la disminución del consumo del cemento, generando así, menor contaminación al medio ambiente.

La resistencia a la compresión del concreto con un $5 \%$ de cenizas térmicas durante los 28 días de curado son los resultados más óptimos, debido a que contribuyen mejor en las propiedades mecánicas del concreto.

A causa de la constante evolución y crecimiento del CM, se está considerando necesario que se empiece a elaborar materiales de concreto con adiciones de residuos agroindustriales, ya que, también traería como beneficio la disminución de los problemas ambientales.

\section{REFERENCIAS}

Adinna, B. O., Nwaiwu, C. M., y O Igwagu, C. J. (Junio de 2019). Effect of rice-husk-ash admixture on the strength and workability of concrete. Nigerian Journal of Technology, 38(1), 4. doi:10.4314/njt.v38i1.7

Adjei, S., y Elkatatny, S. (Diciembre de 2020). A highlight on the application of industrial and agro wastes in cement-based materials. Journal of Petroleum Science y Engineering., 195, 1-14. doi:10.1016/j. petrol.2020.107911 
Aggarwal, V., Gupta, S., y Sachdeva, S. (Diciembre de 2015). Concreto con alto volumen de ceniza volante. Revista Internacional de Ciencias de la Tierra, 2(9). Recuperado el 27 de Mayo de 2021

Águila, I., y Sosa, M. (Diciembre de 2008). Evaluación físico-química de cenizas de cascarilla de arroz; bagazo de caña y hoja de maíz y su influencia en mezclas de mortero; como materiales puzolánicos. Revista Facultad de Ingenieria UCV, 23(4). Recuperado el 21 de Mayo de 2021

Aizpurúa, L., Moreno, G., y Caballero, K. (Octubre de 2018). Estudio del concreto de alta resistencia con el uso de cenizas de materiales orgánicos y polímeros. I+D Tecnológico, 14(2), 29-37. doi:10.33412/idt. v14.2.2071

Alengaram, U., Mo, K., Jumaat, M., Yap, S., y Lee, S. (Marzo de 2016). Green concrete partially comprised of farming waste residues: a review. Journal of Cleaner Production, 117, 122-138. doi:10.1016/j.jclepro.2016.01.022

Ashraf, T., ySI, D. (2020). Mechanical properties of high strength concrete that replace cement partly by using fly ash and eggshell powder. International Conference of Sustainable Earth Resources Engineering. Malasia. doi:10.1016/j.pce.2020.102942

Athira, G., Bahurudeen, A., y Vishnu, V. (Febrero de 2021). Quantification of geographical proximity of sugarcane bagasse ash sources to ready-mix concrete plants for sustainable waste management and recycling. Waste Management and Research, 39(2), 279-290. doi:10.1177/0734242X20945375

Athira, V., Charitha, V., Athira, G., y Bahurudeen, A. (Marzo de 2021). Agro-waste ash based alkali-activated binder: Cleaner production of zero cement concrete for construction. Journal of Cleaner Production, 286, 2. doi:10.1016/j.jclepro.2020.125429

Badiale Furlong, E., Badiale Furlong, V., Kupski, L., Scaglioni, P., Denardi de Souza, T., y Christ Ribeiro, A. (Febrero de 2020). Use of natural resources from Southern Brazil as a strategy to mitigate fungal contamination. Critical Reviews in Food Science y Nutrition, 61(2), 3. doi:10.1080/10408398.2020.17268 68

Ballesteros, C., Serrano, M., y Pérez, D. (Abril de 2019). Respuesta termográfica de bloques de concreto para uso no estructural preparados con ceniza industrial. Revista Industrial, 22(2), 7-26. doi:10.15381/idata. v22i2.15079

Bassam A., T., Rayed, A., Hisham, A., y Abdulaziz, A. (2020). Recycling of rice husk waste for a sustainable concrete: A critical review. Journal of Cleaner Production, 312. doi:10.1016/j.jclepro.2021.127734

Berenguer, R., Lima, N., Cruz, F., Pinto, L., y Lima, N. (Agosto de 2021). Thermodynamic, microstructural and chemometric analyses of the reuse of sugarcane ashes in cement manufacturing. Journal of Environmental Chemical Engineering, 9(4). doi:10.1016/j. jece.2021.105350

Bheel, N., Jokhio, M. A., Abbasi , J. A., Lashari, H. B., Qureshi, M. I., y Qureshi, A. S. (Abril de 2020). Rice Husk Ash and Fly Ash Effects on the Mechanical Properties of Concrete. Engineering, Technology y Applied Science Research, 10(2), 5. doi:10.48084/etasr.3363

Cabrera, M., Sánchez Rodríguez, A., y Aballí Altamirano, C. (Febrero de 2015). Diseño de un sistema para el aprovechamiento integral de la cáscara de arroz. Revista de Ingenieria Energetica, 36(1), 50-60. Recuperado el 24 de Mayo de 2021

Camargo, N., y Higuera, C. (Octubre de 2017). Concreto hidráulico modificado con sílice obtenida de la cascarilla del arroz. Ciencia e Ingeniería Neogranadina, 27(1), 91-109. doi:10.18359/rcin.1907

Canul, J., Moreno, E., y Mendoza, J. (Diciembre de 2016). Efecto de la ceniza volante en las propiedades mecánicas de concretos hechos con agregado calizo triturado de alta absorción. Revista Alconpat, 6(3), 235247. doi:10.21041/ra.v6i3.150 
Celik, K., Meral, C., y Monteiro, P. (Febrero de 2015). Mechanical properties, durability, and life-cycle assessment of self-consolidating concrete mixtures made with blended portland cements containing fly ash and limestone powder. Cement y Concrete Composites, 56, 59-72. doi:10.1016/j.cemconcomp.2014.11.003

Charitha, V., Athira, V., Jittin, V., Bahurudeen, A., y Nanthagopalan, P. (Mayo de 2021). Use of different agro-waste ashes in concrete for effective upcycling of locally available resources. Construction y Building Materials, 285, 3-5. doi:10.1016/j. conbuildmat.2021.122851

Clark, M., Despland, L., Lake, N., Yee, L., Anstoetz, M., Arif, E., y Doumit, P. (Abril de 2017). Highefficiency cogeneration boiler bagasse-ash geochemistry and mineralogical change effects on the potential reuse in synthetic zeolites, geopolymers, cements, mortars, and concretes. Heliyon, 3(4). doi:10.1016/j. heliyon.2017.e00294

D., G., D. Marcon, N., C., E., A., S., y G. A., C. (2020). Statistical analysis of properties of high-volume fly ash concretes as cement replacement. HOLOS. doi:10.15628/ holos.20209805

Da Silva, E., Basto, P., Araujo, F., Miranda, L., y Neto, A. (2019). Evaluation by XRD analysis of the influence of grinding process in the pozolanic activity of sugar cane bagasse ash. Revista Materia, 24(4). doi:10.1590/ s1517-707620190004.0822

Elías, J., Sichez, J., y Reyna, C. (Enero de 2019). Reutilización de plástico pet, papelybagazo de caña de azúcar, como materia prima en la elaboración de concreto ecológico para la construcción de viviendas de bajo costo. Pueblo Continente, 30(1), 169-187. doi:10.22497/PuebloCont.301.30116

Fang-Chen, L., Ming-Gin, L., y Shang-Lien, L. (2020). Effect of coal ash and rice husk ash partial replacement in ordinary Portland cement on pervious concrete. Construction and Building Materials, 286. doi:10.1016/j. conbuildmat.2021.122947
Farnaz, B., Arjumend, M., y Mehmood, A. (2020). Characterization of Sugarcane Bagasse Ash as Pozzolan and Infuence on Concrete Properties. Arabian Journal for Science and Engineering, 45, 10. doi:10.1007/s13369019-04301-y

Fernández, A., y Palomo, Á. (Diciembre de 2015). Propiedades y aplicaciones de los cementos alcalinos. Revista Ingeniería de Construcción, 24(3), 213-232. doi:10.4067/ S0718-50732009000300001

Fuentes, N., Fragozo, I., y Vizcaino, L. (Setiembre de 2015). Residuos agroindustriales como adiciones en la elaboración de bloques de concreto no estructural. Ciencia e Ingeniería Neogranadina, 25(2), 99-116. doi:10.18359/rcin.1434

Gonzáles, C., Montaño, Á., y Castro, D. (Abril de 2015). Obtención y caracterización de geopolímeros, sintetizados a partir de ceniza volante y piedra pómez, utilizados para el desarrollo y mejoramiento del concreto. El Hombre y la Máquina(38), 5965. Recuperado el 21 de Mayo de 2021

Hernández Olivares, F., Medina Alvarado, R., Burneo Valdivieso, X., y Zúñiga Suárez, A. (Junio de 2020). Short sugarcane bagasse fibers cementitious composites for building construction. Construction and Building Materials, 247, 829-837. doi:10.1016/j. conbuildmat.2020.118451

Herrmann, A., Koenig, A., y Dehn, F. (Junio de 2018). Structural concrete based on alkali activated binders: Terminology, reaction mechanisms, mix designs and performance. Structural Concrete, 19(3), 918-929. doi:10.1002/suco.201700016

Huaquisto, S., y Belizario, G. (Junio de 2018). Utilización de la ceniza volante en la dosificación del concreto como sustituto del cemento. Revista de Investigaciones Altoandinas, 20(2), 225-234. doi:10.18271/ ria.2018.366

Izquierdo, I. S., Izquierdo, O. S., y Ramalho, M. (Diciembre de 2018). Physical and mechanical properties of concrete using 
residual powder from organic waste as partial cement replacement. Construction Engineering Magazine, 33(3), 12. doi:10.4067/S0718-50732018000300229

Jagadesh, P., Ramachandramurthy, A., y Murugesan, R. (Octubre de 2018). Overview on properties of sugarcane bagasse ash (SCBA) as Pozzolan. Indian Journal of Geo-Marine Sciences, 47(10), 1934-1945. Recuperado el 22 de Abril de 2021

Jannat, N., Hussien, A., Abdullah, B., y Cotgrave, A. (Setiembre de 2020). Application of agro and non-agro waste materials for unfired earth blocks construction: A review. Construction y Building Materials, 254, 1-27. doi:10.1016/j.conbuildmat.2020.119346

Katare, V. D., y Madurwar, M. V. (Setiembre de 2019). Design and investigation of sustainable pozzolanic material. Journal of Cleaner Production, 242, 9. doi:10.1016 / j.jclepro.2019.118431

Katare, V., y Madurwar, M. (Julio de 2021). Process standardization of sugarcane bagasse ash to develop durable highvolume ash concrete. Journal of Building Engineering, 39, 3. doi:10.1016/j. jobe.2021.102151

Krishna, N. K., Sandeep, S., y Mini, K. M. (2016). Study on concrete with partial replacement of cement by rice husk ash. IOP Conference Series: Materials Science and Engineering, (pág. 12). doi:10.1088/1757899X/149/1/012109

Mattey, P., Robayo, R., Díaz, J., y Delvasto, S. (Enero de 2015). Aplicación de ceniza de cascarilla de arroz obtenida de un proceso agro-industrial para la fabricacion de bloques en concreto no estructurales. La Revista Latinoamericana de Metalurgia y Materiales, 35(2), 285-294. Recuperado el 21 de Mayo de 2021

Memon, M., Jhatial, A., Murtaza, A., Raza, M., y Phulpoto, K. (Marzo de 2021). Production of eco-friendly concrete incorporating rice husk ash and polypropylene fibres. Environmental Science and Pollution Research. doi:10.1007/s11356-021-13418-3
Minnu, S., y Bahurudeen, A. (30 de Octubre de 2020). Comparison of sugarcane bagasse ash with fly ash and slag: An approach towards industrial acceptance of sugar industry waste in cleaner production of cement. Journal of Cleaner Production, 285(20), 124836. doi:10.1016/j. jclepro.2020.124836

Mishurov, N., Voytyuk, M., Vinogradov, P., Machneva, O., y Voytyuk, V. (Abril de 2021). Application of waste products of crop processing in the production of building materials for agricultural facilities. IOP ConferenceSeries: Earth and Environmental Science, 723, págs. 2-6. Moscow. doi:10.1088/1755-1315/723/3/032053

Mohd Arif, S., Rokiah, O., Khairunisa, M., Chong, W. B., Chek, C. Y., Youventharan, D., y Doh, S. I. (Octubre de 2021). Compressive strength of concrete containing eggshell powder aspartial cement replacement. IOP ConferenceSeries: Earth and Environmental Science, 682, pág. $15 . \quad$ Putrajaya. doi:10.1088/1755-1315/682/1/012031

Montiel Bohórquez, N., y Pérez, J. (Febrero de 2019). Generación de Energía a partir de Residuos Sólidos Urbanos. Estrategias Termodinámicas para Optimizar el Desempeño de Centrales Térmicas. Información Tecnológica., 30(1), 273-283. doi:10.4067/S0718-07642019000100273

Msinjili, N. S., Schmidt, W., Rogge, A., y Kühne, H. C. (Agosto de 2019). Rice husk ash as a sustainable supplementary cementitious material for improved concrete prop. African Journal of Science, Technology, Innovation and Development, 11(4), 10. doi :10.1080/20421338.2018.1513895

Nagajyothi, K., Shyam Chamberlin, K., Asadi, S. S., y Maheswara Reddy, M. (Diciembre de 2019). Experimental study on strength and workability of concrete using rice husk ash and quarry dust concrete. Ravinder Kumar Targotra et al. International Journal of Recent Research Aspects, 6(4), 35-41. Recuperado el 20 de Mayo de 2021 
Oliko, C., Kabubo, C. K., y Mwero, J. N. (2020). Rice straw and eggshell ash as partial replacementsof cement in concrete. Engineering, Technology y Applied Science Research, 10(6), 7. doi:10.48084/etasr.3893

Peñaranda Gonzalez, L., Montenegro Gómez, S., y Giraldo Abad, P. (Setiembre de 2017). Aprovechamiento de residuos agroindustriales en Colombia. Revista de Investigación Agraria y Ambiental., 8(2), 141-150. doi:10.22490/21456453.2040

Prakash, R., Thenmozhi, R., y Raman, S. (2020). Hormigón reforzado con fibra que contiene agregado de cáscara de coco de desecho, cenizas volantes y fibra de polipropileno. Revista Facultad de Ingenieria Universidad de Antioquia. (94), 33-42. doi:10.17533/10.17533/udea. redin.20190403

Praveenkumar, T. R., Vijayalakshmi, M. M., y Meddah, M. S. (2019). Strengths and durability performances of blended cement concrete with. Construction and Building Materials, 9. doi:10.1016 / j.conbuildmat.2019.05.045

Rajasekar, A., Arunachalam, K., Kottaisamy, M., y Saraswathy, V. (2018). Durability characteristics of Ultra High Strength Concrete with treated sugarcane bagasse ash. Construction and Building Materials, $171, \quad 7$. doi:10.1016/j. conbuildmat.2018.03.140

Robayo, R., Mattey, P., y Delvasto, S. (Noviembre de 2015). Comportamiento mecánico de un concreto fluido adicionado con ceniza de cascarilla de arroz (CCA) y reforzado con fibras de acero. Revista de la construcción, 12(2). doi:10.4067/S0718915X2013000200011

Rodier, L., Villar Cociña, E., Ballesteros, J., y Holmer Savastano, J. (Setiembre de 2019). Potential use of sugarcane bagasse and bamboo leaf ashes for elaboration of green cementitious materials. Journal of Cleaner Production, 231, 54-63. doi:10.1016/j. jclepro.2019.05.208
Ruiz, A., Peñaranda, C., Fuentes, G., y Semprun, M. (Mayo de 2020). Análisis comparativo de resultados en el uso de la ceniza de bagazo de caña de azúcar como material sustituyente del cemento portland en el concreto. Sostenibilidad, Tecnología y Humanismo,11(2), 8-17. doi:10.25213/22161872.51

Šadzevi ius, R., Gurskis, V.,y Ramukevi ius, D. (Enero de 2015). Sustainable construction of agro-industrial buildings from straw panels. Proceedings of the International Scientific Conference: Rural Development., 1-6. doi:10.15544/RD.2015.012

Sathvik, S., Edwin, A., Basnett, A., Sharma, P., y Carmicheal, J. (2019). Experiment of partial replacement of egg shell powder and coconut fibre in concrete. International Journal of Innovative Technology and Exploring Engineering (IJTTEE), 8(6S4), 10341038. doi:10.35940 / ijitee.F1213.0486S419

Scope, C., Vogel, M., y Guenther, E. (Abril de 2021). Greener, cheaper, or more sustainable: Reviewing sustainability assessments of maintenance strategies of concrete structures. Sustainable Production and Consumption, 26, 838-858. doi:10.1016/j.spc.2020.12.022

Serag, F., Sahar A., M., Bassam A., T., y Taher A., T. (2021). The effect of using nano rice husk ash of different burning degrees on ultrahigh-performance concrete properties. Construction and Building Materials, 290. doi:10.1016/j.conbuildmat.2021.123279

Shakouri, M., Exstrom, C. L., Ramanathan, S., y Suraneni, P. (2020). Hydration, strength, and durability of cementitious materials incorporating untreated corn cob ash. Construction and Building Materials, 11. doi:10.1016/j.conbuildmat.2020.118171

Silva, Y., y Delvasto, S. (Mayo de 2015). Concreto Autocompactante con diferentes niveles de ceniza volante y escoria de combustión de carbón. Revista Colombiana de Materiales(5), 1-9. Recuperado el 27 de Mayo de 2021 
Souza, A., Ferreira, H., Vilela, A., Viana, Q., Mendes, J., y Mendes, R. (Octubre de 2021). Study on the feasibility of using agricultural waste in the production of concrete blocks. Journal of Building Engineering, 42. doi:10.1016/j.jobe.2021.102491

Tan, Y. Y., Doh, S. I., y Chin, S. C. (2018). Eggshell as a partial cement replacement in concrete development. Magazine of Concrete Research, 9. doi:10.1680 / jmacr.17.00003

Tariq, A., Abdullah, S., Daddan Khan, B., Abdul Salam, B., y Zaheer, A. (2021). Mechanical and Durability Properties of Aerated Concrete Incorporating Rice Husk Ash (RHA) as Partial Replacement of Cement. Crystals, 11(604). doi:10.3390/ cryst11060604

Tiong, H. Y., Lim, S. K., Lee, Y. L., Yew, M. K., y Lim, J. H. (2020). Absorption and strength properties of lightweight foamed concrete witheggshellpowderaspartialreplacement material of cement. 2nd International Conference on Civil y Environmental Engineering, 476, pág. 012021. doi:10.1088 / 1755-1315 / 476/1/012021

Torres de Sande, V., Sadique, M., Pineda, P., Bras, A., Atherton, W., y Riley, M. (2021). Potential use of sugar cane bagasse ash as sand replacement for durable concrete. Journal of Building Engineering, 39(102277). doi:10.1016/j.jobe.2021.102277
Townsend, S., Spreadbury, C., Laux, S., Ferraro, C., Kari, R., y Townsend, T. (Setiembre de 2020). Blending as a Strategy for Reusing Municipal Solid Waste Incinerator Ash in Road-Base Construction. Journal of Environmental Engineering., 146(9), 1-9. doi:10.1061/(ASCE)EE.1943-7870.0001788

Vargas, Y., y Pérez, L. (Marzo de 2018). Aprovechamiento de residuos agroindustriales para el mejoramiento de la calidad del ambiente. Facultad de Ciencias Básicas, 14(1), 59-72. Recuperado el 21 de Mayo de 2021

Venkata, R., Dayakar, P., y Mohan, S. J. (Julio de 2019). Effect of egg shell powder on strength behaviour of concrete. International Journal of Innovative Technology and Exploring Engineering, 8(9S3), 3. doi:10.35940/ijitee. I3110.0789S319

Zareei, S., Ameri, F., y Bahrami, N. (Setiembre de 2018). Microstructure, strength, and durability of eco-friendly concretes containing sugarcane bagasse ash. Construction and Building Materials, 184, 258 - 268. doi:10.1016/j. conbuildmat.2018.06.153 